\title{
Observational properties of visual binaries as modelled using a synthetic catalogue ${ }^{\star}$
}

\section{For visual apparent magnitudes $m_{v}<15$}

\author{
P. Nurmi ${ }^{1}$ and H. M. J. Boffin ${ }^{1,2, \star \star}$ \\ 1 Royal Observatory of Belgium, Av. Circulaire 3, 1180 Brussels, Belgium \\ 2 European Southern Observatory, Karl-Schwarzschild-str. 2, 85748 Garching-bei-München, Germany
}

Received 31 January 2003 / Accepted 4 June 2003

\begin{abstract}
A simulated binary star catalogue is created that represents the full sky distribution of stars, excluding those with galactic latitude $|b|<10^{\circ}$, down to $m_{\mathrm{v}}<15$. The catalogue is compatible with observed magnitude and number distributions of the HST Guide Star Catalog. The simulated catalogue is analyzed for observational properties of visual binary stars, both in a general context without taking any observational constraints into account, and using different observability criteria that model the observational capability of the astrometric DIVA satellite. It is shown that several observational bias effects should be taken into account when final output catalogues are analyzed. The results indicate that $\Delta m_{\mathrm{v}}$ distribution peaks at $\Delta m_{\mathrm{v}} \approx 7$ and to maximize the number of binary detections with large mass ratios, the magnitude threshold should be set as high as possible. This is especially important if low mass $M<0.2 M_{\odot}$ secondaries are studied. The predicted number of optical pairs is calculated and for large magnitude differences close to the galactic plane and toward the galactic center it may be difficult to discriminate between a red companion and a low-mass foreground/background star for distant double stars for which no parallax measurements are available. Using optimal detection procedures for optical double stars it is estimated that, depending on the assumed double star distributions, 60\%-70\% of all the visual binary stars in the catalogue with separations $0.0625^{\prime \prime}<\theta<3.5^{\prime \prime}$, $m_{\mathrm{v}}$ (secondary) $<22$, and $\Delta m<10$ could be observed during the DIVA or similar missions.
\end{abstract}

Key words. methods: data analysis - stars: binaries: general - stars: binaries: visual

\section{Introduction}

To optimize the forthcoming astrometric missions in their capabilities to detect different types of binary stars requires detailed preliminary planning. One way to do this is to create an artificial catalogue that has statistically similar properties to the expected final output catalogue. This allows us to do pre-launch fine-tuning of the methods and procedures used in the final data reduction of forthcoming astrometric satellites. Also, some other binary studies using already existing catalogues may benefit from the results of this kind of analysis. With this aim in mind, we create an artificial binary ${ }^{1}$ catalogue that represents the overall distributions of binary stars in the sky down to visual magnitude $m_{\mathrm{v}}=15$. This limit is

\footnotetext{
Send offprint requests to: P. Nurmi, e-mail: Pasi .Nurmi@oma . be

* Appendix A is only available in electronic form at

http://www . edpsciences.org

$\star \star$ e-mail: Henri.Boffin@oma.be

1 Throughout the paper we use the word "binary" to describe a physical system while by double star we refer to a system that can be a physical (i.e. gravitationally bound) or an optical double.
}

chosen so that it represents the observational limit of the DIVA satellite and it is also, roughly, the completeness limit of the source catalogue used, GSC 1.2 (the enhanced version of the Guide Star Catalog 1.2; see Morrison et al. 2001) from which initial apparent magnitude distributions are taken. We should note that after the submission of this article all further development of the DIVA mission was stopped and DIVA is now definitely canceled. Our results will, however, still be useful for future similar missions but, more importantly, they represent a first necessary exploratory step in addressing the possibilities of the GAIA astrometric mission with regards to the detection of binaries.

Our approach differs from traditional star count models (e.g. Bahcall \& Soneira 1980; Gilmore \& Reid 1983; Bahcall $\&$ Soneira 1984 , etc.) by the fact that we use an existing source catalogue directly as our observational basis and the galaxy model is considered only when proper weights in the final distribution of binary properties are taken into account. In this study we consider only relative numbers with respect to the total number of binary stars assumed to exist for all the stars in the sky down to $m_{\mathrm{v}} \approx 15$. Absolute numbers could be 
calculated if the numbers given in the paper are multiplied by the overall binary fraction $f_{\text {binary }}$ and the total number of stars $N_{\text {tot }}$. According to Duquennoy and Mayor (1991) $f_{\text {binary }}$ is $\sim 0.6$ for G-dwarf field stars, but it may be different for cluster stars and different spectral types (Duchêne et al. 2001). Using star counts from the GSC 1.2 catalogue and considering only those objects having non-star classification, the absolute number of stars can be estimated to be $\sim 2.1 \times 10^{7}$ stars in the whole sky. This can be compared with the galaxy model prediction by Bahcall \& Soneira (1980) that gives $3.7 \times 10^{7}$ stars when $m_{\mathrm{v}}<15$. The difference is mainly due to the incompleteness of GSC and the fact that some non-star classification objects are in fact close-by stars.

\section{The input for the binary catalogue}

Our approach is to create an artificial binary catalogue that is compatible with

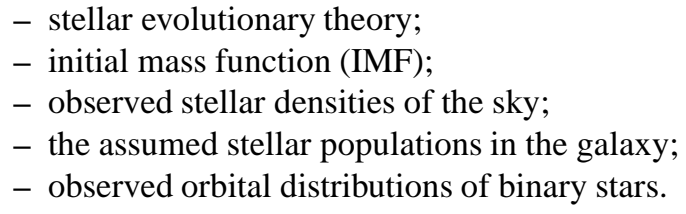

To fulfill these requirements we have used a hybrid approach in the binary catalogue construction. The main idea is to start from the IMF and to evolve stars according to their age and metallicity distributions and place them at random distances from the Earth. Then by varying the distance and stellar properties we find a match to the observed $m_{\mathrm{v}}$ distribution of GSC catalogue and repeat the whole procedure for every individual field and then collect the information covering the whole sky. In the simulations typically $\sim 10^{3}$ random fields are chosen each having a size of $\sim 1$ square degree. Thus, in the final sample we have $\sim 4 \times 10^{5}$ binary stars, sufficient for our statistical analysis. However, it should be noted that since we use random distances and weights in the final analysis, the number of hypothetical stars that would be required to produce similar results using the galaxy model directly is much larger by several orders of magnitude.

\subsection{Initial stellar distributions and GSC 1.2 source catalogue}

In the simulation we use the GSC 1.2 catalogue as an input catalogue to have a correct magnitude distribution and stellar density in each sample of the sky. The nominal magnitude limit of the catalogue is 15.5 , but it is not fully complete down to this limit. To evaluate the completeness of the GSC we have estimated the completeness by calculating the magnitude $m_{\text {limit }}$ at which $m_{\mathrm{v}}<m_{\text {limit }}$ for $90 \%$ of the stars in $10^{3}$ random fields: $m_{\text {limit }}$ corresponds roughly to the turnover point of the cumulative distribution of stars. The results are averaged over the galactic longitude $l$ and they are shown as a function of ecliptic latitude $b$ (Fig. 1). It is evident that the completeness limit is much smaller at low galactic latitudes. Since it is only close to the galactic plane that the completeness is not so good, we

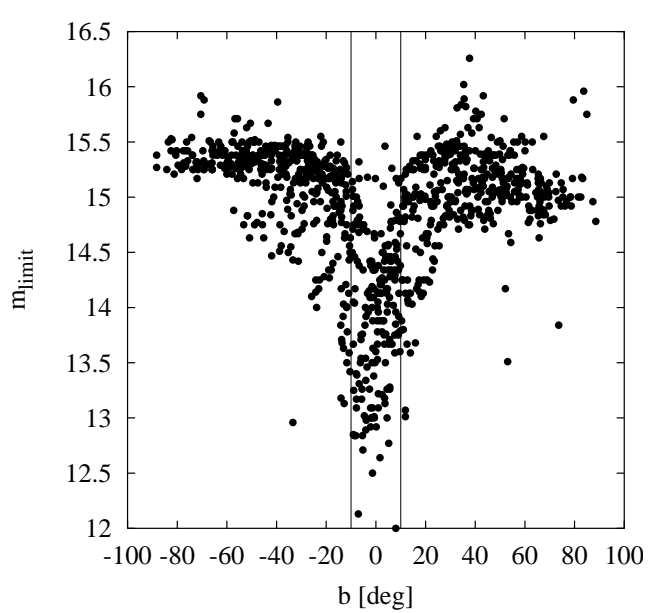

Fig. 1. The magnitude limit of the cumulative distribution where $90 \%$ of objects have $m_{\mathrm{v}}<m_{\text {limit }}$ as a function of $b$ averaged over $l$. The zone of avoidance in the calculation is located between the two vertical lines.

have not included any corrections for this, since this has no effect on our statistical results. This was also confirmed by doing a smaller scale simulation using a collection of GSC 2.2 fields where the catalogue is complete to much larger magnitudes than that of the GSC 1.2.

For our purpose the GSC 1.2 represents the full-sky distribution of stars well enough so that it gives suitable observational data for the magnitude distribution during the magnitude fitting. In GSC 1 mainly two band-passes are used in the plates: photographic $V$ (north) and photographic $J$ (south) and short exposure $V$ plates are used in the galactic plane. Using the colour equation from Tritton (1983), $J=V+0.72(B-V)$, and assuming that the difference between photographic $V$ and Johnson $V$ are small, we have changed $V$ and $J$ magnitudes to Johnson $V$ bandpass. This approximation is good enough for our purpose, because we are only concerned with a magnitude precision of $0.5 \mathrm{mag}$. Since the colour is known from the simulations, all the magnitudes are scaled to $m_{\mathrm{v}}$ using colour conversion equations given in Caldwell et al. (1993), where coefficients of polynomial fits for different colour conversions are given. Since we transform GSC magnitudes to $m_{\mathrm{v}}$ and use this magnitude in our synthetic catalogue we need not to convert from $m_{\mathrm{v}}$ back to GSC photometric magnitudes.

The overall magnitude errors of the catalogue are a few tens of magnitudes. For our purpose this accuracy is high enough, since we are only interested in statistical averages of the simulated catalogue. The only requirement in the fitting procedure is that the magnitude histogram is accurate enough at the one magnitude level.

The digital resolution of the scanning is $1.7^{\prime \prime}$, and all the binaries in our simulations that have separations $\theta$ less than this are considered unresolved and their combined magnitude is used in the fitting procedure. For resolved binaries we use the magnitude of the brighter star.

To calculate the observed $m_{\mathrm{v}}$-distribution, we select a large number of fields in the sky that are transformed into galactic coordinates $l_{\mathrm{c}}$ and $b_{\mathrm{c}}$, where "c" denotes the central position of the field. A selected field is a circle with radius of $30^{\prime}$. 
The sample size should be large enough so that the surface stellar density variations over the sky are well sampled, but making the field size larger does not bring any additional information. The observed $m_{\mathrm{v}}$-distribution is then calculated from this list of stars after the conversion, explained above, is applied to the GSC magnitudes.

\subsection{Stellar evolution}

\subsubsection{IMF}

There is strong evidence that initial masses of stars in different birthplaces all derive from the same initial mass distribution (Kroupa 2002). In our simulations, individual masses of stars are chosen from the initial mass function that is taken from Kroupa (2002). By using the observed star count data and stellar luminosity functions Kroupa et al. (1993) derived the IMF that is corrected for unresolved binaries. They assumed the binary fraction to lie between 0.6 and 0.7 and that binaries are derived from the same IMF as a result of random pairing. The derived mass function for single stars is a multi-part power-law having turn-off points at $0.5 M_{\odot}$ and at $1 M_{\odot}$. Brown dwarfs are not included in the simulation.

The detailed shape of the distribution and the coefficients of the power-law are given in the supplementary material of the Kroupa (2002) article.

Since the agreement about the IMF is not complete we have also used the IMF of Miller and Scalo (1979) and the observed present day mass function (PDMF) obtained for low-mass stars by Kroupa et al. (1990), but it turns out that the results remain roughly the same. This is mainly due to the fact that functions differ mainly in their low and far ends that are not statistically important in the final observed distributions. The masses used in the simulations are between $0.08 M_{\odot}$ and $100 M_{\odot}$. These are also the limits of applicability of Hurley et al. (2000) stellar evolution algorithms.

However, it is not clear how binaries should be formed from the IMF or if a general IMF for binaries does exist at all. In principle there are three different possibilities to create a binary from the initial IMF. The most simple way, as used in the IMF derivation obtained by Kroupa et al. (1993), is just to choose two random masses from the same IMF and create a pair. Another way is to choose a combined mass from the IMF and use some distribution for the mass ratio $Q=m_{2} / m_{1}\left(m_{1}\right.$ is the mass of the heavier component and $m_{2}$ is the lighter secondary component) to calculate $m_{2}$. Further, the first mass could be obtained from the IMF and then calculate the secondary mass from the $Q$ distribution. Since there is a great discrepancy about the actual form of the $Q$-distribution and how it may depend on the spectral type, we use the two first ways to create a binary although the second way violates the assumptions behind the initial IMF derivation. Nevertheless, it is interesting to see how the results change if it is used. If we calculate the $Q$-distribution by choosing two masses randomly from the IMF, for three different cases IMFs, shown by three histograms together with the approximated combined smooth distribution, distributions are very similar (Fig. 2, dot-dashed line). This can be compared with the observed mass-ratio distribution of nearby G-dwarf

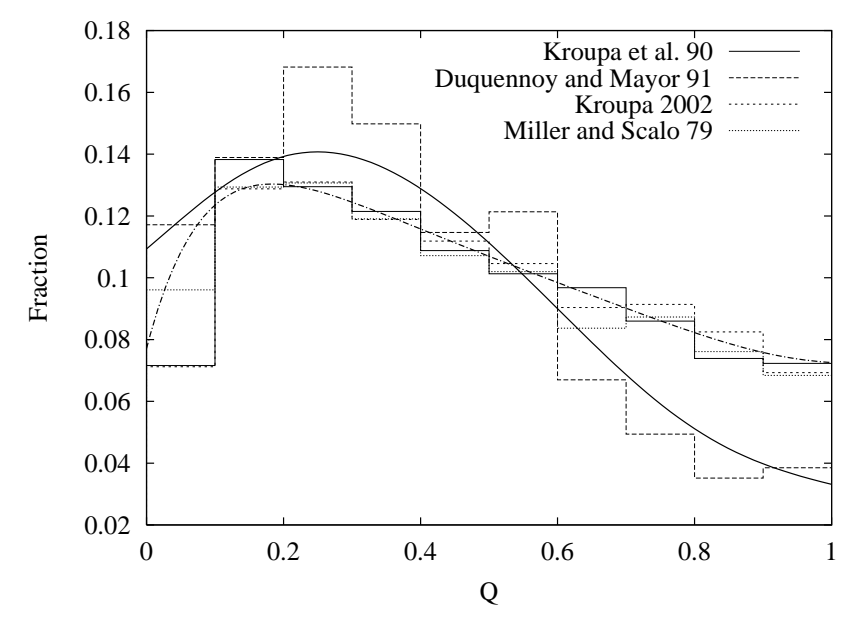

Fig. 2. The mass ratio $Q$-distributions as derived from three different IMF (Miller \& Scalo 1979; Kroupa et al. 1993; Kroupa 2002) and the observed $Q$-distribution of G-dwarf stars (Duquennoy \& Mayor 1991).

stars (histogram and the smoothed distribution, shown in Fig. 2 as a solid line). It is understandable that the compatibility is not very good, since the observed $Q$-distribution is for G-type primaries. As we notice later the PDMF corresponds closely to IMF for low-mass stars and therefore it has no influence on the discrepancy.

\subsubsection{Star formation rate}

Although some studies find evidence of non-uniform star formation rates (see, e.g., the review by Rana 1991), for simplicity, we have used a uniform star formation rate in all the simulations. For disk stars we assume that the star formation rate has been uniform between $10 \mathrm{Myr}$ and $10 \mathrm{Gyr}$, and for halo stars between 10 Gyr and 16 Gyr.

\subsubsection{Evolutionary tracks}

Since we start our calculation from the IMF, we have to evolve stars to derive present-day mass and luminosity functions. Stellar evolution is done using the analytical stellar evolution equations given in Hurley et al. (2000). As an input we give the present epoch $t_{\mathrm{f}}$ to which the stars are evolved, initial masses of stars $m_{\mathrm{i}}$ and metallicities $Z$. As an output we obtain present-day masses $m_{\mathrm{f}}$, effective temperatures $T_{\text {eff }}$ and absolute bolometric magnitudes $M_{\mathrm{b}}$. In the final output catalogue we have several different types of stars including main-sequence stars, giants and white dwarfs. No mutual interaction or mass-transfer between the two stars is included.

\subsection{Galactic distribution of stars}

Since we are interested only in stars having $m_{\mathrm{v}}<15$ we can use a rather general model for the galactic stellar density. Galactic disk component clearly dominates the number distributions for this magnitude limit even at high galactic latitudes (see, e.g., $\mathrm{Ng}$ et al. 1997). This was also confirmed by varying galaxy 
model parameters during the analysis and the results remain the same, except for some extreme unrealistic values.

Due to the poor determination of extinction and the resulting large uncertainty of the galaxy models close to the galactic plane, we have excluded all stars that have $|b|<10^{\circ}$. In our catalogue, the galaxy model is taken into account by introducing weights to different stars according to their distances from the earth. The weights are related to stellar density functions that are calculated from Bahcall (1986) where all the functions and model parameters are given. Since we use the existing source catalogue we do not use absolute numbers of stellar counts, but instead the weights are used only to correct distance distributions of stars. The absolute numbers of stars in a certain direction $(l, b)$ are taken directly from the GSC catalogue.

\subsubsection{Disk}

The galactic disk density law is a double exponential where the Galactocentric distance of the Earth is $8.5 \mathrm{kpc}$ and the height above the plane is $27 \mathrm{pc}$ with local normalization of 0.13 stars per $\mathrm{pc}^{3}$. The scale length is $3.5 \mathrm{kpc}$ and for mainsequence (MS) stars the scale height depends on the absolute magnitude, $M_{\mathrm{abs}}: h_{z}=82.5 \times M_{\mathrm{abs}}-97.5$ (Bahcall $\&$ Soneira 1980). For giants the scale length is constant: $h_{z}=250 \mathrm{pc}$. Uniform star formation rate between $10 \mathrm{Myr}$ and $10 \mathrm{Gyr}$ is assumed. Metallicity $[\mathrm{Fe} / \mathrm{H}]$ is taken from the age-metallicity relationship given in Rana (1991) and the metallicity distribution is modelled as a Gaussian with $\sigma=0.2$ and $[\mathrm{Fe} / \mathrm{H}]$ depends on $t_{\mathrm{f}}($ Fan 1999).

\subsubsection{Halo}

The halo is modelled as a deprojected de Vaucouleurs profile (Bahcall 1986). The flattening factor is 0.8 and the local normalization is $C=0.00026$ stars per $\mathrm{pc}^{3}$. As for the disk, the star formation rate is assumed to be constant, but the population II stars are generally much older and the age is randomly distributed between 10 Gyr and 16 Gyr. The age-metallicity relationship for halo stars is the same as in $\mathrm{Ng}$ et al. (1997) and the metallicities obtained are between 0.0004 and 0.003 .

\subsection{Binary distributions}

The orbital parameters of binaries have been chosen from the following distributions:

- Inclination $i$ : uniform in $\cos (i)$.

- Eccentricity $e$ : in model I, $f(e)=2 e$ (i.e. the distribution that is expected in the case of thermalized orbits due to passing stars, Hills 1975) and in model II, we use the multipart distribution for the eccentricity as given in Duquennoy \& Mayor (1991). This distribution is derived for G-dwarf stars and may not be valid for other spectral types, but since they dominate our sample, we have used it as a second option. When the orbital period $P<11.6 \mathrm{~d}$, orbits are circular and in the intermediate range, $11.6 \mathrm{~d}<P<1000 \mathrm{~d}, f(e)$ is Gaussian with $\sigma=0.15$ and $\bar{e}=0.3$. When $P>1000 \mathrm{~d}$ $f(e)=2 e$.
- Semi-major-axis $a$ : in model $\mathrm{I}, \log (a)$ is assumed to be uniformly distributed between $0.1 \mathrm{AU}$ and $10^{6} \mathrm{AU}$ (Öpik 1924). In model II, $\log (P)$ has a Gaussian distribution with $\sigma=0.23$ and $\overline{\log (P)}=4.8$ (Duquennoy \& Mayor 1991).

- Remaining angular elements $\omega, \Omega$ and $M$ have a uniform distribution between 0 and $2 \pi$.

- Mass-ratio $Q$ : in model a, we choose $m_{1}$ and $m_{2}$ independently from the same IMF. In model $b$, the total mass $m_{\text {tot }}=m_{1}+m_{2}$ is chosen from the IMF and $m_{2}$ is calculated using the $Q$-distribution taken from Duquennoy and Mayor (1991).

Hence, we have four different models $\mathrm{I}_{\mathrm{a}}, \mathrm{I}_{\mathrm{b}}$ and $\mathrm{II}_{\mathrm{a}}, \mathrm{II}_{\mathrm{b}}$. In the results we show complete distributions only for $I_{a}$ and for other models we describe how they differ from the first model.

\subsection{Simulation algorithm}

Simulation is started by choosing central coordinates for the field that has a size $A_{\text {field }}$. All the objects from the GSC 1.2 catalogue entering the field are obtained and from these only objects having a star classification are taken. This underestimates the absolute numbers of stars, since the objects with non-star classification may be either close-by stars or galaxies. Since we exclude stars situated at the galactic plane where most nonstar classification objects are star-star pairs, and at high galactic latitudes non-star objects are most likely galaxies, we are not introducing any crucial bias to the results. Then the apparent visual magnitude distribution is calculated for this sample.

After the observational part, the visual magnitude of the trial star $m_{\mathrm{v}}$ is calculated from

$m_{\mathrm{v}}=M_{\mathrm{v}}+5 \log \left(\frac{r}{10 \mathrm{pc}}\right)+A_{\mathrm{v}}$,

where the distance $r$ is taken from a uniform distribution, the absolute visual magnitude $M_{\mathrm{v}}$ is obtained from the IMF via stellar evolution algorithms and the extinction $A_{\mathrm{v}}=3.1 E(B-V)$ is obtained from the colour excess $E(B-V)$ that is calculated according to the extinction model given by Chen et al. (1999) that should be a good approximation when $|b|>2.5^{\circ}$. The colour is obtained using $T_{\text {eff }}$ and the data by Flower (1996), where empirical tables of $T_{\text {eff }}$ and $B-V$ colours are given. Also, the bolometric correction $B C$ is calculated according to Flower's data using the polynomial fits to the tables. The previous steps are repeated to obtain a visual magnitude also to the secondary star. After visual magnitudes have been obtained for both components we generate the binary elements and calculate the visual separation $\theta$. If $\theta>1.7^{\prime \prime}$ we use the primary magnitude in the fit and in the case of unresolved binary star we use the combined magnitude. The procedure is repeated until we have a match between the trial star and a star in the observed $m_{\mathrm{v}}$ distribution with the overall accuracy of $\pm 0.5 \mathrm{mag}$. In this way we fit stellar magnitude distributions in our artificial catalogue to the observed stellar distributions and new stars are chosen from the initial sample until all stars in the field have a match in the distribution. Then a new field is chosen and the match in magnitude distribution is obtained in the same way as before.

The catalogue created in the previous way must be corrected since initially the distance is taken randomly from the 


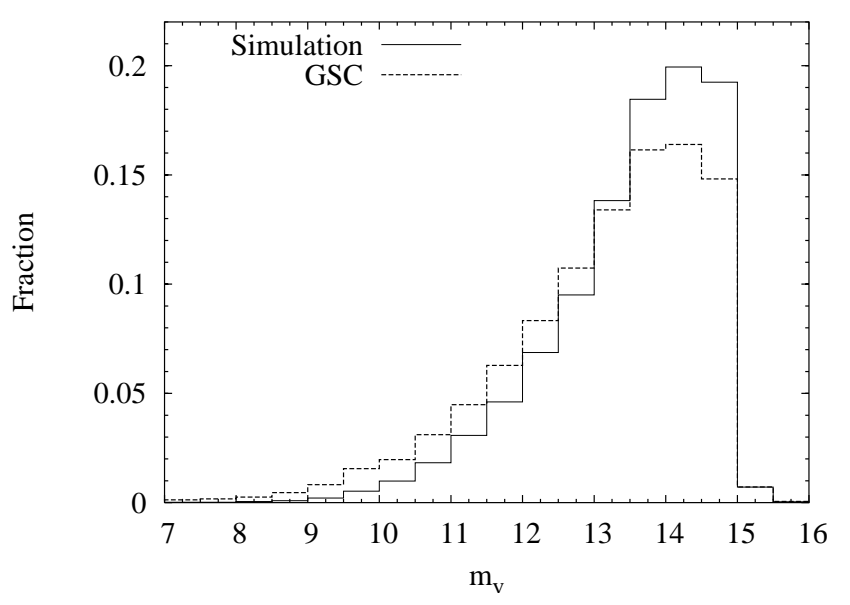

Fig. 3. GSC visual magnitude distribution (GSC) as obtained directly from the input catalogue is shown together with the $m_{\mathrm{v}}$-distribution taken from the simulation. The simulated distribution deviates systematically from the GSC distribution. This is corrected by $w_{j}^{2}$ correction weights.

uniform distribution. The correction is done by weighting every star by the weight $w_{i}^{1}$, that depends on the galactic stellar density (calculated using Bahcall 1986 equations) and $r^{2}$ since the volume element toward a certain direction $\mathrm{d} V=\omega r^{2} \mathrm{~d} r$, where $\omega$ is the solid cone angle and $r$ is the distance from the Earth. We have chosen to use weights just from the practical point of view, since the fitting procedure is much faster and it is easy to test different galaxy models and the sensitivity of the results to different parameters by using this approach and not to choose stars from the real distributions originally.

However, it is not obvious that the magnitude distribution in the catalogue and the original magnitude distributions are the same after the weight is added to the simulations. The scaled distributions would be exactly the same only if $\sum w_{i}^{1}=f_{m_{j} \pm \mathrm{d} m}$, where $f_{m_{j} \pm \mathrm{d} m}$ is the fraction of stars in the original GSC distribution in the interval $m_{j} \pm 0.25$ in the $j$ th bin. The index $i$ goes from 1 to the maximum number of stars in the corresponding magnitude interval. Since in every magnitude bin there are samples of stars from different distances, the previous condition is roughly true. In Fig. 3, we show the original GSC magnitude distribution together with the distribution obtained from the simulation. We see that there is a systematic change in the new distribution so that at small magnitudes, the number of stars is underestimated while at large magnitudes, it is overestimated. To make sure that this does not cause any distortion to our results, we have corrected for this effect. This is done by introducing a new weight $w_{j}^{2}$ that is the ratio between the observed and simulated magnitude distributions. In the final analysis we multiply all the stars having a magnitude $m$ in the interval $m_{j} \pm 0.25$ with the corresponding additional weight $w_{j}^{2}$. This guarantees that the final magnitude distribution agrees perfectly with the GSC distribution. We emphasize that the usage of the additional weight does not change the overall distance distribution of stars and therefore the influence of the new weight is very small in the final binary distributions.

To make sure that different parts of the simulation have been randomized enough, we run the simulation a few times
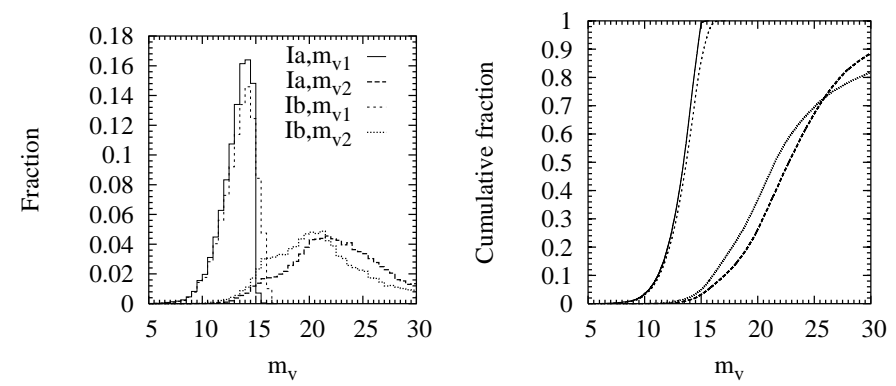

Fig. 4. Visual magnitude distribution of primary $m_{\mathrm{v} 1}$ and secondary $m_{\mathrm{v} 2}$ (left panel) and corresponding cumulative distributions (right panel).

using different fields and initial conditions, but it turns out that the results remain always the same. Hence, the errors of this study come mainly from the initial conditions and assumptions used in the simulations and not from the Poisson errors associated to Monte Carlo simulations.

\section{The analysis of the catalogue}

\subsection{General properties}

In this section we give statistical distributions of the whole catalogue without considering any observational effects. These results represent "true" binary distributions if all of them would be observed. All real observational projects or astrometric satellites sample only a small fraction of these populations, depending on the observational capabilities of the missions.

For visual binaries there are three main observables $m_{\mathrm{v} 1}$, $m_{\mathrm{v} 2}$ and $\theta$ that observations should be able to provide. In Fig. 4 (left panel) histograms of $V$-band magnitudes for $m_{\mathrm{v} 1}$ (brighter component) and $m_{\mathrm{v} 2}$ are given for Ia and Ib models. Also, cumulative distributions are given (right panel) to make it easy to derive the fractions of detected stars in different magnitude limits. The primary distribution cuts sharply at $m_{\mathrm{v}} \approx 15$ due to the completeness limit of the GSC and the overall differential magnitude count distribution $\mathrm{d} \Phi\left(m_{\mathrm{v}}\right)$ for $m_{\mathrm{v} 1}$ is presented well by the distribution $\mathrm{d} \Phi\left(m_{\mathrm{v}}\right) \propto 10^{\lambda m_{\mathrm{v}}} \mathrm{d} m_{\mathrm{v}}$, where $\lambda=0.25 \pm 0.03$. This distribution is the global average over the whole sky and $\lambda$ is different in different parts of the sky. Secondary distributions are very wide and they peak at $m_{\mathrm{v}}=21$ and $m_{\mathrm{v}}=22$ for models b and a, respectively. Only $5 \%$ of $m_{\mathrm{v} 2}$ have $m_{\mathrm{v}}<15$. In model $b$, there are more stars having smaller magnitudes, since the mass ratio distributions are different.

When we calculate magnitude difference distributions for Ia and Ib we see that $\Delta m$ has its maximum between 6 and 8 and that after $\Delta m=3$ the distribution increases quickly (Fig. 5). In model $b$, the distribution is narrower and drops much more quickly after the maximum. The location of the $\Delta m$ maximum value follows mainly from the mass-ratio distribution and the mass-luminosity relationship.

Logarithmic separation, $\log \theta$, distributions are shown in Fig. 6, for models I and II. Only model a is used, since a and $\mathrm{b}$ give similar results. The distribution of model $\mathrm{I}$ is flat up to $\sim 3^{\prime}$, a direct result of the initial $\log a$ distribution. Beyond this the binary frequency drops smoothly and practically no 

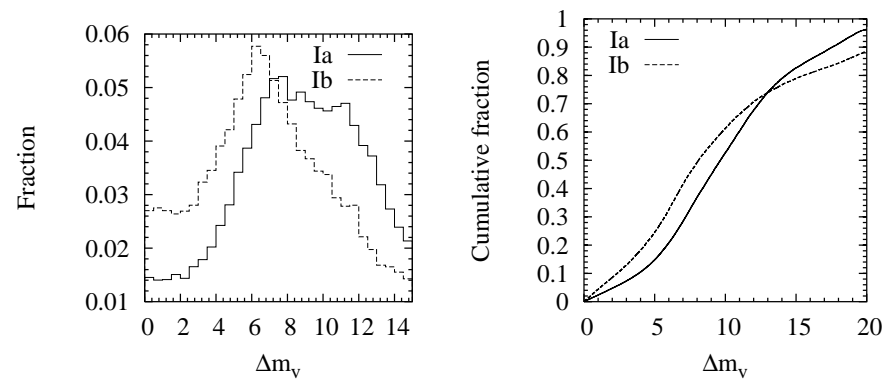

Fig. 5. Magnitude difference distributions of binaries for models Ia and Ib (left panel) and corresponding cumulative distributions (right panel).
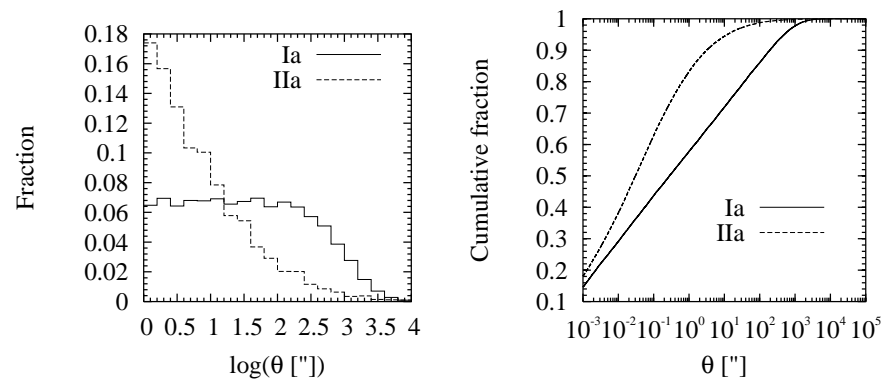

Fig. 6. Mutual separation distribution of binary systems (left panel) and corresponding cumulative distribution (right panel).

binaries exist with $\theta>50^{\prime}$. A gradual decrease in the distribution follows from the used upper limit for semi-major axes $\left(a_{\max }=10^{6} \mathrm{AU}\right)$, that causes the upper limit to $\theta$ and distance beyond which all binaries are unresolved. Model II distribution is very different from that of model I. There are fewer binaries having large separations compared to close-by systems. Cumulative distributions for both models are shown in the right panel and from this we note that half of the objects have $\theta<1^{\prime \prime}$ in model I and $75 \%$ in model II.

\subsubsection{Temperature}

Since we have detailed information of the individual stars we can also calculate the colour $B-V$ histograms and effective temperature histograms for both components (Figs. 7 and 9, left panels). Secondaries are typically much redder than primary stars since fainter stars are typically M-type stars, but brighter stars are most likely G-type dwarfs. In the whole sample the most likely distance is $\sim 700 \mathrm{pc}$, but the tail extends to a few kilo-parsecs. If only resolvable binaries are included the distance distribution is naturally very different, since in this distribution the brightness is dominated by primaries that are in unresolved systems. Color and distance distributions are very similar for all models, the only difference being that there are more blue secondary stars in model $b$ than in model a. The difference between models $a$ and $b$ is more obvious in effective temperature plot (Fig. 9, left panel). In model b, the G-type secondary population is larger.
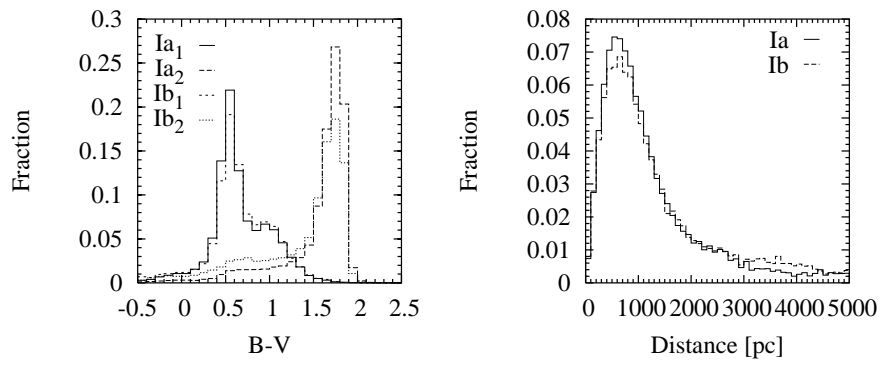

Fig. 7. Color $B-V$ distributions for primary and secondary stars (left panel) and distance distribution of binaries (right panel). Models $\mathrm{Ia}$ and $\mathrm{Ib}$ are used in the plots.

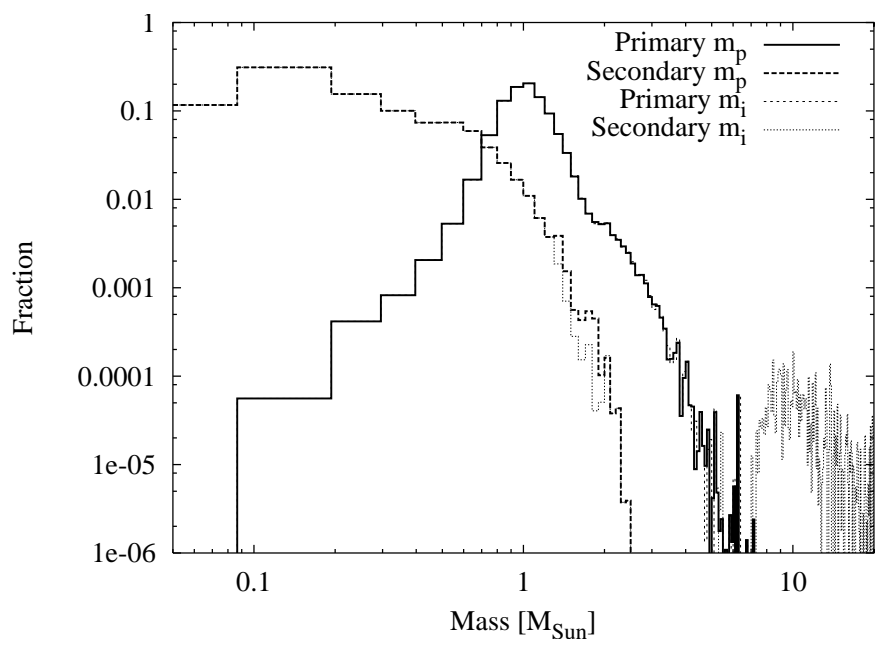

Fig. 8. Initial and present-day mass distributions for both components in model Ia. One solar mass stars dominate the primary distribution and $0.1 M_{\odot}$ star dominates the secondary distribution. Large mass star $M>5 M_{\odot}$ have evolved to white dwarf secondaries.

\subsubsection{Masses}

In Fig. 8, initial $\left(m_{\mathrm{i}}\right)$ and present-day mass $\left(m_{\mathrm{p}}\right)$ distributions are given for both components (model Ia). The primary mass distribution peaks at $1 M_{\odot}$ and the most common mass for the secondary is $\sim 0.1 M_{\odot}$. Large mass stars with $M>5 M_{\odot}$ have evolved to white dwarf secondaries that can be seen in the initial mass distribution in the right part of the figure. The initial mass distribution of primaries remains almost unchanged. Mass distributions of model $b$ differs from the shown histogram, but looks qualitatively similar and is therefore not shown.

The mass ratio distributions for models Ia and IIb are plotted in Fig. 9 (right panel) together with the calculated distributions using Eq. (4). The distirbution of model II differs clearly from the one of model I and the calculated distribution does not represent the observed G-type binary distribution very well. If we restrict the sample to G-type stars within $200 \mathrm{pc}$ from the Earth (Fig. 10, right panel), then the fit is better for model b, but for model a the differences are large, when $Q<0.2$. The agreement between Duquennoy and Mayor (1991) distribution and model $\mathrm{b}$ is expected, since it was used to generate the initial $Q$ distribution. In model a, the peak at $0.1<Q<0.2$ comes from the IMF that increases toward small masses and if we would 

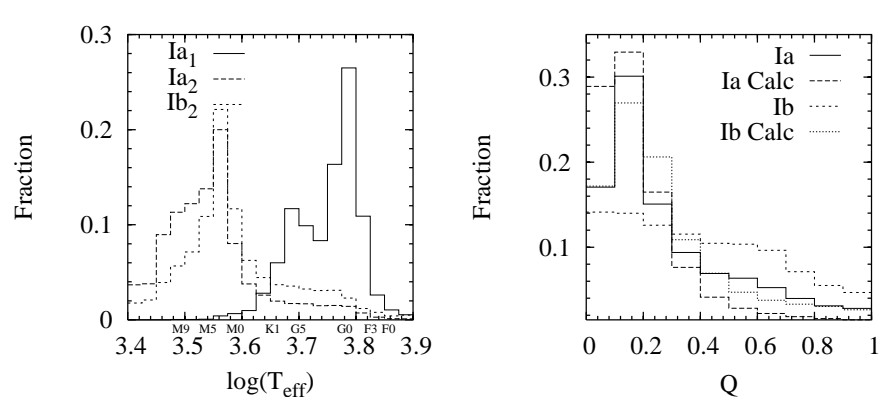

Fig. 9. Effective temperature (and spectral type) distributions of all the stars in the catalogue (left panel). Secondary star distributions are shown for models a and $\mathrm{b}$. In the right panel, mass ratio $Q$ distributions for models Ia and IIb catalogue binaries are given together with the $Q$ distribution calculated using the Eq. (4).
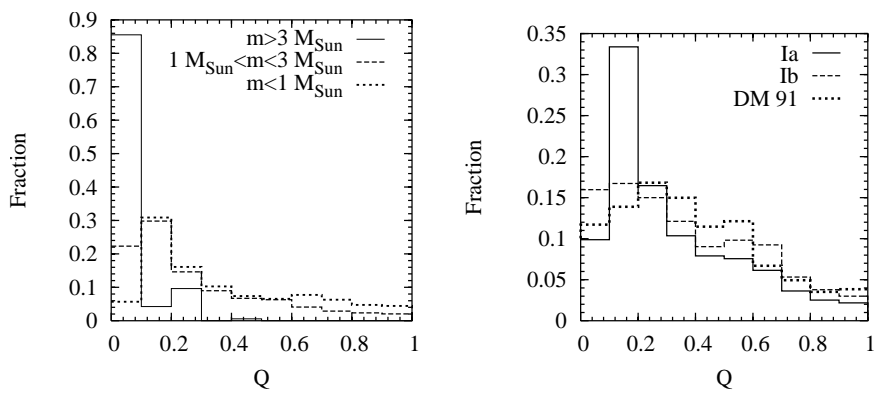

Fig. 10. In the left panel, the $Q$-distribution is shown for three different primary mass intervals (model Ia). In the right panel, we have compared the observed G-dwarf $Q$-distribution to the ones derived from the model Ia and Ib simulations (only G-type stars are included that have distances $d<200 \mathrm{pc}$ ).

not restrict our initial masses to $M>0.08 M_{\odot}$, also the last interval $0<Q<0.1$ would be much higher.

To see how the $Q$-distribution changes as a function of primary mass $M_{1}$ we have taken three different mass intervals $\left(M_{1}>3 M_{\odot}, 1 M_{\odot} \geq M_{1} \leq 3 M_{\odot}\right.$ and $\left.M_{1}<1 M_{\odot}\right)$, in which the $Q$-distribution is calculated separately (Fig. 10, left panel). Since we are interested in qualitative change, we use only model Ia stars. As expected, the distribution is much more flat for small masses, a fact that can cause bias effects in some restricted binary samples.

\subsubsection{Barycentric radial velocities of the components}

Intrinsic tangential and radial velocities of the components are also of interest for astrometric and radial velocity missions. To evaluate the intrinsic radial velocity distributions in our catalogue we have calculated the barycentric velocity components for both stars. Tangential velocities are typically very small, generally less than $10^{-2}$ arcsec per year and for a typical binary star the intrinsic tangential motion will not be observed.

Radial velocities can be very large and for distant unresolved binaries it can be the only observational method for vrad.epsbinary detection. Radial velocity distribution is peaked close to zero and the distribution is naturally symmetric with respect to the zero axis. A good knowledge about the distributions can be obtained when the cumulative distributions are calculated. The cumulative curves for both binary components

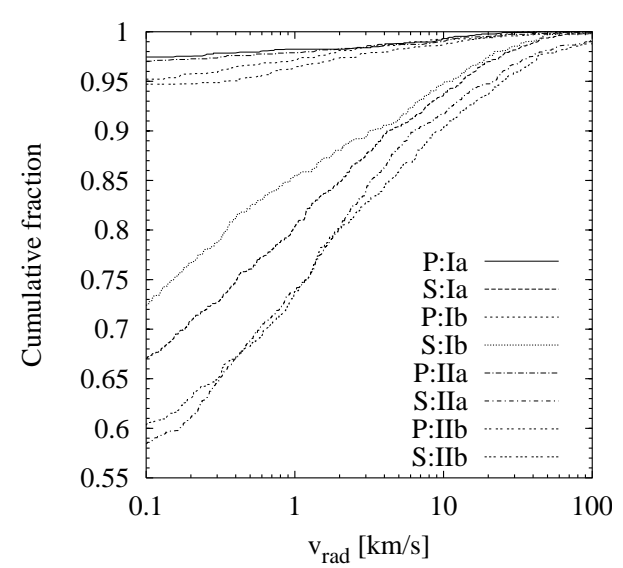

Fig. 11. Cumulative radial velocity distributions for both binary components given to four different models.

are shown in Fig. 11. We emphasize that the Galactic velocity component is ignored, since our galaxy model is static.

\subsubsection{Luminosity class}

Main sequence binaries where both components are still in the main-sequence is the largest population in all the models. Their fraction is 0.68 in model a and 0.65 in model $\mathrm{b}$. The second largest group includes systems where the primary is evolved away from the MS. Their fractions are 0.27 and 0.24 for models a and b, respectively. Also an interesting sub-group is a pair where the primary is in the main-sequence and the secondary is a white dwarf. Their fraction is 0.05 in model a and 0.11 in model $b$. The magnitude differences are generally much larger than for MS+MS binaries. The $\Delta m$-distribution starts at $m_{\mathrm{v}}=19$ and peaks at $m_{\mathrm{v}}=26$. Hence, a typical magnitude difference between a primary and a white dwarf secondary is $\sim 11$ mag. The typical colour for a white dwarf is generally bluer than that of the M-type stars. These facts makes their detection very difficult, especially for DIVA, where the CCD is more sensitive to red stars. The results derived to white dwarfs should be taken only as a first approximation, since no mass exchange or any other physical interaction between the components is included. Better modelization of binaries is required if specific close binaries are to be included in the analysis.

\subsection{Diva observations of the visual binaries}

In principle, visual double star detection procedures can be divided into two different categories depending on $\Delta m$ and $\theta$. If $\Delta m$ is small $(<4)$ and $\theta$ is $<1^{\prime \prime}$, then the PSF fitting of the images may show that PSF is a composite of two PSFs. If $\Delta m$ is large and the separation is well beyond the central PSF it may happen that no direct detection of the secondary is available, but by co-adding all the images at different transits much fainter background objects can be revealed than would be possible otherwise. We have developed an efficient procedure that can be used in the image combination for observations containing binned pixels and rotated images. The principles of this procedure are given in Appendix A. 
In DIVA, CCDs pixels are binned into $1 \times 4$ pixels in crossscan direction, the pixel scale being $0.25^{\prime \prime} / \mathrm{pix} \times 1^{\prime \prime} /$ pix. Once the images are combined, the initial field of view $\left(3^{\prime \prime} \times 7^{\prime \prime}\right)$ becomes circular with a radius of $\sim 3.5^{\prime \prime}$. This is the outer limit in our calculations. The inner limit is set to $1 / 4$ of the pixel size i.e. $\theta_{\min }=0.0625^{\prime \prime}$. Beyond the outer limit, only those double stars that are bright enough and generate individual windows, can be detected. Their number is statistically very small (see Figs. 4 and 6) and they are not considered here, although some of these can be very interesting objects, since accurate orbits could be calculated.

We model the observational capability of DIVA when the image combination procedure is used by writing that the double star is resolvable if

$\log \Delta \theta>a \Delta m+b$,

where $\Delta \theta$ is the separation in arcseconds and $\Delta m$ is the difference in magnitude. The coefficients $a$ and $b$ depend on the procedure by which the analysis is made. For image combining, the estimated optimal values are: $a=0.25$ and $b=-2$. Also a limiting magnitude must be estimated below which the signal-to-noise ratio is too small for detection. Based on our preliminary tests, we define the following limiting magnitudes for the detection: if $\theta>1.5^{\prime \prime}$ then $m_{\lim }=22$ and when $\theta \leq 1.5^{\prime \prime}$ then $m_{\lim }=c \log \theta+d$, where $c=-5.5$ and $d=23$. In reality all the coefficients depend on the number of transits, colours of the objects and CCD properties. The previous coefficients are for the most common case, for which the number of images is 142 and the secondary star is a red M5-type star. Also, optimal observing conditions are assumed.

Using the previous conditions we have calculated the fractions of stars that are observed in the whole synthetic catalogue and show the results in $\Delta m$ and $\theta$ bins for direct evaluation of the observational capabilities. By "observed" we mean that the stars fullfill the previous conditions for $\Delta \theta$ and $\Delta m$ as $m_{\lim }$ and $\theta$ limits are fixed. All fractions are numbers of "observed" binaries divided by the total number of binaries in the whole catalogue. We show only the histograms for model Ia, since otherwise the plots would be too confused.

In Figs. 12 and 13, four curves are given, where "Combining" means that we have used the previous conditions directly and in " $m_{\text {lim }}=20$ " we have set $m_{\lim }=20$, but otherwise the conditions are the same. The third curve (PSF fitting) is for conservative PSF fitting estimation, where we still use the previous conditions, but we set $m_{\mathrm{lim}}=19.5$ and $\Delta m_{\max }=5$. The last (All) is without DIVA conditions considering all the binaries with $\Delta m<10, m_{\mathrm{lim}}=22$ and $0.0625^{\prime \prime}<\theta<3.5^{\prime \prime}$. The plots are different for every model, but model Ia gives the smallest values and model IIb gives the highest values. The curves for models IIa and Ib are located between these two extremes, in this order. The integrated values of the $\Delta m$ and $\theta$ distributions for all the models are given in Table 1, where we also give values for all stars that have $0.0625^{\prime \prime}<\theta<3.5^{\prime \prime}\left(\mathrm{All}_{2}\right)$ or $\theta<3.5^{\prime \prime}\left(\mathrm{All}_{3}\right)$. The completeness in each $\Delta m$ or $\theta$ bin for different observability criterias can be calculated if the corresponding values at the Table 1 are divided by the values from the "All" column. The largest incompleteness is for small separations $\theta<0.5$ " and for large magnitude differences $\Delta m>5$.
Table 1. Fractions of binaries in four different detection categories. The last two columns are for the whole catalogue when $0.0625^{\prime \prime}<$ $\theta<3.5^{\prime \prime}\left(\mathrm{All}_{2}\right)$ and when $\theta<3.5^{\prime \prime}\left(\mathrm{All}_{3}\right)$.

\begin{tabular}{ccccccc}
\hline \hline Model & Comb & $m_{\text {lim }}=20$ & PSF $_{\text {fit }}$ & All & $\mathrm{All}_{2}$ & $\mathrm{All}_{3}$ \\
\hline $\mathrm{I}_{\mathrm{a}}$ & 0.061 & 0.050 & 0.036 & 0.11 & 0.25 & 0.66 \\
$\mathrm{I}_{\mathrm{b}}$ & 0.088 & 0.075 & 0.057 & 0.13 & 0.25 & 0.66 \\
$\mathrm{II}_{\mathrm{a}}$ & 0.079 & 0.066 & 0.049 & 0.14 & 0.33 & 0.90 \\
$\mathrm{II}_{\mathrm{b}}$ & 0.11 & 0.094 & 0.074 & 0.17 & 0.33 & 0.90 \\
\hline
\end{tabular}

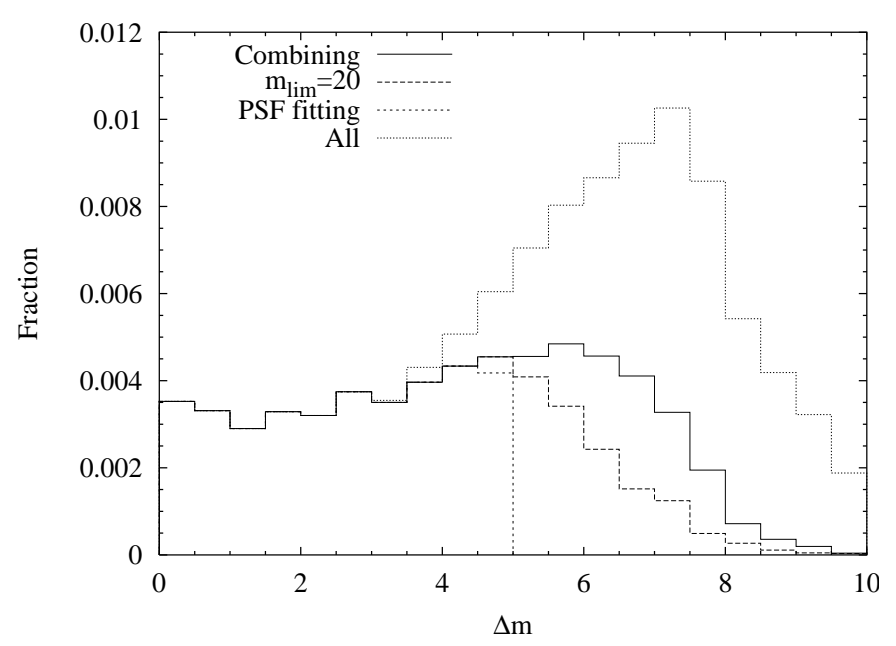

Fig. 12. Fractions of binaries having different observability criteria. Combining means that we use Eq. (2) together with $m_{\text {lim }}$ condition given in the text. For $m_{\text {lim }}=20$ we have used this as a maximum $m_{\mathrm{v}}$ that could be detected from the images and for, PSF fitting, we set $m_{\mathrm{lim}}=19.5$ and $\Delta m$ maximum to 5 mag. For "All" we restrict the sample to $0.0625^{\prime \prime}<\theta<3.5^{\prime \prime}, m_{\mathrm{v}}$ (secondary) $<22$, and $\Delta m<10$ without considering any other conditions.

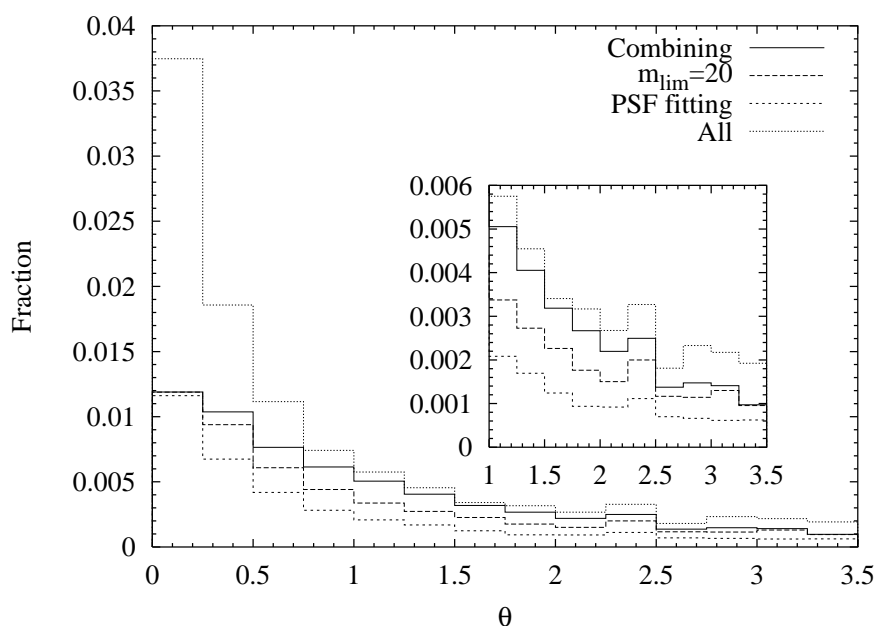

Fig. 13. Same as Fig. 12, but for 8 different separation bins.

Using the image combining method the completeness is between $60 \%$ and $70 \%$ depending on the model. The image combination is an important step to detect large magnitude differences and it can provide a considerable amount of new double star detections that could not be possible otherwise. 


\section{Properties of "detected" binaries}

\subsection{Statistical analysis of random pairing}

In the final analysis of binarity in the output catalogue it is important to be able to make a distinction between binaries and optical double stars. For DIVA this distinction can be difficult to do if $\Delta m$ is large and no spectral information for the secondary or the primary from the dispersed images is available, since for DIVA the sky mapper images are obtained in white light. In these cases we can only calculate the expected number of background/foreground stars in the field that depend on the average stellar density down to the magnitude of the secondary star. Statistically we have $\bar{N}$ background stars in the field, with $\bar{N}=A_{\text {field }} n_{l, b}\left(m<m_{l}\right)$, where $A_{\text {field }}$ is the size of the field and $n_{l, b}\left(m<m_{l}\right)$ is the surface number density of stars having $m<m_{l}, m_{l}$ denoting the limit down to which stars are calculated and $l, b$ are the galactic coordinates. In a typical case, the field of view is small and $\bar{N} \ll 1$, therefore we have to calculate the probability that we have $N_{b}$ background stars in the field. If we assume that this follows roughly a Poisson distribution (see e.g. Scott and Tout 1989), then the probability of having $N_{b}$ background stars in the field is simply

$P=\frac{\bar{N}^{N_{b}}}{N_{b} !} \exp (-\bar{N})$.

Respectively, $1-P\left(N_{b}=0\right)$ gives the probability for having at least one optical double star in the field.

To evaluate the expected number of optical doubles we have calculated the probabilities using the Bahcall and Soneira (1986) model for stellar density predictions in three different ecliptic longitudes as a function of ecliptic latitude (Fig. 14). To estimate the number of stars that are slightly below the maximum detection threshold $m_{\mathrm{v}}(\max )=22$ we have used $m_{\mathrm{v}}=23$ in the calculations. The field used in the calculations is a circle with a radius of $3.5^{\prime \prime}$ i.e. the one corresponding to the DIVA observational field. Restricting the sample to only double stars having $\theta<3.5^{\prime \prime}$ we exclude $34 \%$ (in the models Ia and IIa) or $10 \%$ (in the models Ib and IIb) of all the binaries in our catalogue.

The results can be extrapolated to smaller magnitude limits by dividing the values by 1.4 per magnitude. This estimation is based on the cumulative number distribution given by galaxy model predictions. Hence, only for small galactic latitudes $|b|<20^{\circ}$ and toward the galactic center is there a considerable number of false doubles in the field. Beyond this, the probability is very small, of the order of $10^{-2}$.

\subsection{Physical properties}

If we have observations of only one colour or white light observations and the only observables are $m_{1}, \Delta m$ and $\theta$, then we can have only a little additional information about the physical properties of the bodies. Some additional assumptions can allow us to derive more information. If we assume that the general form of the mass-luminosity relation is $L \propto M^{K}$ and suppose that both bodies are on the main sequence, then

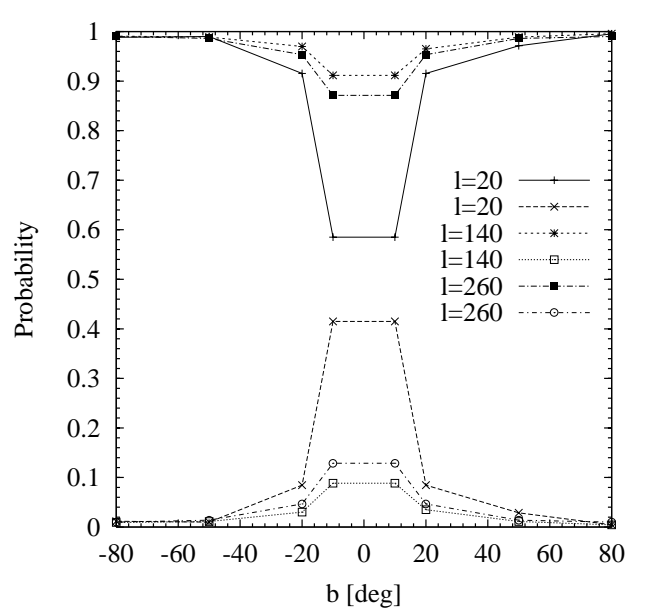

Fig. 14. The three upper curves give the probabilities of having no background stars down to $m_{\mathrm{v}}=23$ in combined DIVA field as a function of galactic latitude for three different galactic longitudes. The three lower curves give the probabilities of having at least one optical double star in the field.

we can calculate the mass ratio directly from the magnitude difference

$\log \left(M_{a} / M_{b}\right)=0.4 \Delta m / \kappa$.

Equation (4) applied to our catalogue gives a rough estimation for the $Q$ distribution. We have used $\kappa=4$ in the calculation (Fig. 9, right panel). However, there is clearly a trend that the equation underestimates mass ratios at large values and overestimate $Q$ at small values.

In addition, if we know spectral types of the stars and parallax estimations we can calculate absolute magnitudes to the components. This can give us the upper limit to the age of the system if the primary is a massive star and hence exclude the ambiguity for the secondary to be either a low mass MS star or a white dwarf, even if the spectral type of the secondary is not known. For bright binaries, parallax and proper motion measurements can resolve the background star ambiguity if the measurements are accurate enough. If a binary is a nearby system we might see a trail in the combined image revealing the motion of the secondary. However, the fraction of these objects is very small, but they might be interesting cases for later binary studies, since the orbital elements could probably be determined.

\section{Discussion}

Our synthetic catalogue and the analysis of double star observations include several approximations. Especially important to the output is the way binaries are assumed to form. The results in this study agree with the results of Duquennoy \& Mayor (1991). In a recent study, Halbwachs et al. (2003) found that the $Q$-distribution is very different from the Duquennoy \& Mayor (1991) distribution, but only for binaries with $P<50$ days. This does not change our results, since only a very small fraction of binaries in our sample have $P<50$ days (7\% in models I and 9\% in models II). Also, we believe that the uncertainties associated with the galaxy model or binary distributions 
are not that severe and the results represent statistically correct binary distributions. A separate but closely related topic is to estimate the number of astrometric binaries and how these overlap with visual binaries. It is important to know how different types of binary detection methods are complementary and how the global binary analysis should be done. This is closely related to the details of astrometric missions and is out of the scope of this study.

In the future we will do similar studies, but applicable to GAIA, using a more accurate galaxy model and including binary evolution in the simulations. This step is crucial to GAIA, since the magnitude limit is much larger. However, some results may not change remarkably for visual binaries since the resolvability criteria restricts the sample within a few kpc. Since multiband photometry is done by GAIA, much better physical information can be obtained for both components and better estimations for binary properties of the system can be made.

Acknowledgements. This project was supported by the ESAPRODEX contract "From HIPPARCOS to GAIA" with No. $14847 / 00 / \mathrm{NL} / \mathrm{SFe}(\mathrm{IC})$. Dr. J. Hurley is gratefully acknowledged for providing the routines of his analytic stellar evolution code and the anonymous referee is acknowledged for valuable comments that led to the improvement of the paper.

\section{References}

Bahcall, J. N., \& Soneira, R. M. 1980, ApJS, 44, 73 Bahcall, J. N., \& Soneira, R. M. 1984, ApJS, 55, 67
Bahcall, J. N. 1986, ARA\&A, 24, 577

Caldwell, J. A. R., Cousins, A. W. J., Ahlers, C. C., van Wamelen, P., \& Maritz, E. J. 1993, S.A.A.O. Circ., 15, 1

Chen, B., Figueras, F., Torra, J., et al. 1999, A\&A, 352, 459

Duchêne, G., Simon, T., Eislöffel, J., \& Bouvier, J. 2001, A\&A, 379, 147

Duquennoy, A., \& Mayor, M. 1991, A\&A, 248, 485

Fan, X. 1999, AJ, 117, 2528

Flower, P. J. 1996, ApJ, 469, 355

Fruchter, A. S., \& Hook, R. N. 2002, PASP, 114, 144

Gilliland, R. L., Nugent, P. E., \& Phillips, M. M. 1999, ApJ, 521, 30

Gilmore, G., \& Reid, N. 1982, MNRAS, 202, 1025

Halbwachs, J. L., Mayor, M., Udry, S., \& Arenou, F. 2003, A\&A, 397, 159

Hills, J. G. 1975, AJ, 80, 809

Hurley, J. R., Pols, O. R., \& Tout, C. A. 2000, MNRAS, 315, 543

Kroupa, P., Tout, C. A., \& Gilmore, G. 1990, MNRAS, 244, 76

Kroupa, P., Tout, C. A., \& Gilmore, G. 1993, MNRAS, 262, 545

Kroupa, P. 2002, Science, 295, 82

Miller, G. E., \& Scalo, J. M. 1979, ApJS, 41, 513

Morrison, J. E., Röser, S., McLean, B., Bucciarelli, B., \& Lasker, B. 2001, AJ, 121, 1752

Ng, Y. K., Bertelli, G., Chiosi, C., \& Bressan, A. 1997, A\&A, 324, 65

Öpik, E. 1924, Publ. de l'Obs. Astron. de l'Univ. de Tartu, t. XXV, No. 6

Rana, N. C. 1991, ARA\&A, 29, 129

Scott, D., \& Tout, C. A. 1989, MNRAS, 241, 109

Tritton, S. 1983, UK Schmidt Telescope Handbook, Royal Observatory, Edinburgh 
P. Nurmi and H. M. J. Boffin: Simulated catalogue of visual binaries. I., Online Material p 1

\section{Online Material}




\section{Appendix A: Double star detections with large magnitude differences by co-adding images at different transits}

If an image combination procedure must be applied to every star, that has typically $\sim 10^{2}$ transits during the astrometric mission with probably several different images from each transit, then the procedure must be simple and fast, while still being able to enhance the resolution and to increase the signalto-noise ratio in the combined image. These requirements in mind we have developed an image combining procedure that is based on the drizzling algorithm developed by Fruchter \& Hook (2002). The drizzling or the method of variable-pixel linear reconstruction is a procedure in which input pixels (for DIVA $1 \times 4$ ) are sub-sampled to some output grid that have smaller $1 \times 1$ pixels (by a fraction $f_{1}$ ) than in the initial frame. Before actual mapping, the initial pixels are shrunk (by a fraction $f_{2}$ ) or some other procedure (e.g. Gaussian weighting) is used so that the final image is not smoothed too much (Gilliland et al. 1999). Different weights $w_{x y}$ can be introduced to individual pixels so that geometrical distortions or any relevant corrections can be taken into account if we have a priori knowledge about them. The kernel of the algorithm, that is repeated until all the images are combined, is

$W_{x y}^{\prime}=a_{x y} w_{x y}+W_{x y}$

$I_{x y}^{\prime}=\frac{a_{x y} i_{x y} w_{x y} s^{2}+I_{x y} W_{x y}}{W_{x y}^{\prime}}$,

where $I_{x y}$ is the combined intensity, $W_{x y}$ is the combined weight, $w_{x y}$ is individual weight of the pixel, $a_{x y}$ is the geometrical weight (overlapping factor), $s$ is the fraction between the size of the output pixel to the input pixel and, finally, $i_{x y}$ is the measured pixel value. In Eqs. (A.1) and (A.2), $x$ and $y$ refer to pixel positions in the input and output grids and dotted values refer to values at the next iteration. The free parameters $f_{1} \leq 1$ and $f_{2} \leq 1$ must be determined from general algorithm tests. The modification of the basic pixel drizzling procedure is related only to the way the geometrical overlappings are calculated. For binned pixels we can either subdivide the pixels back to $1 \times 1$ pixels that are individually shrank or then another possibility is to shrink $1 \times 4$ pixels directly by some fraction before they are mapped to the new grid.

\section{A.1. Different phases in the image combination and object detection}

The main steps in the procedure are:

1. Shift and rotate

2. Choose new background grid by ratio $s=\mathrm{pix}_{\text {out }} / \mathrm{pix}_{\mathrm{in}}$ finer than the grid in the original CCD;

3. Shrink initial pixels to reduce grid convolution effects (by factor $f_{2}$ );

4. Map shrank pixels to underlying grid and add all images together using the modified drizzle algorithm;

5. Create the PSF for the central hypothetical single star using all the information that is available and affects the PSF (i.e. positions, magnitude, colour etc.);
6. Run PSF through the same algorithm using the same $f_{1}$ and $f_{2}$ values as used in the main frame containing additional components;

7. Scale images and subtract the combined PSF from the main frame calculated in step 4;

8. Apply an identification algorithm to the final image to detect faint companions.

The last four steps are required to detect anything with large $\Delta m$ close to the central component and they all are normally performed, since we generally do not know what is hidden in the images.

In an ideal case after the adding procedure the signal-tonoise ratio for $i$ images is

$\frac{S}{N}=\frac{\sum S_{i}}{\sqrt{\sum N_{i}^{2}}}$

The ideal magnitude gain can be easily estimated. If all the images are similar $S_{i}=S_{j}=S$ and only Poisson error is considered, then in $n$ images we get a magnitude threshold higher by

$\Delta m_{\text {gain }}=2.5 \log \sqrt{n}$.

This makes a difference of 1 mag in 6 images, 1.25 for $10,1.85$ for 30 and 2.22 for 60 images. However, in reality, this is not exactly true and especially for faint objects the signal is readout noise limited and then

$\frac{S}{N} \approx \sqrt{n} \sqrt{\left(\frac{S}{1+R^{2} / S}\right)}$,

where $R$ is the readout noise. Hence, the proportional factor is $<2.5$, but the gain is still proportional to $\sqrt{n}$.

In reality the noise behavior is more complicated and the drizzling procedure itself has an effect on the noise since, in adjacent pixels, the noise is correlated. Due to the correlation, the measured noise in a drizzled image is smaller than that obtained by just interlacing the images. A detailed noise behavior depends on $f_{1}, f_{2}$ and overlapping geometry.

\section{A.2. About PSF subtraction}

The main purpose of PSF subtraction is to see faint features around bright objects. To do good PSF fitting and subtraction, high $\mathrm{S} / \mathrm{N}$ is required and good knowledge about the center and the wings of the PSF is required. For a small number of rotations, binning can be a severe problem in the detection capability, since the magnitude gain can be small and unequally distributed outside the central area. If the PSF changes as a function of position on the chip and the spectrum of the target, or if there is any change in the PSF shape in time (focus etc.) that is not known a priori, then subtraction with the "wrong" PSF can create some spurious errors and false detections. One possibility to solve this problem would be to use PSFs obtained at close-by locations and to build up a PSF library from where PSFs can be obtained. Also, simulated PSFs could be used but this does not solve the problem of a PSF evolving with time. 